\title{
Pyran Polymer
}

National Cancer Institute

\section{Source}

National Cancer Institute. Pyran Polymer. NCI Thesaurus. Code C29343.

A hydrocarbon-based polymer containing a pyran nucleus used to bioconjug ate various drugs,. Use of a pyran polymer-drug bioconjug ate may alter the biodistribution of a low molecular weight drug, resulting in disease-specific targeting with reduced drug access to sites of toxicity such as the bone marrow; the large size of the bioconjug ate does not allow the active agent to pass the blood-bone marrow barrier. ( $\mathrm{NCIO4)}$ 